\title{
BMJ Open Lessons learned on the experienced facilitators and barriers of implementing a tailored VBHC model in a Dutch university hospital from a perspective of physicians and nurses
}

\author{
Dane Lansdaal (D) , ${ }^{1}$ Femke van Nassau, ${ }^{2}$ Marije van der Steen, ${ }^{1}$ \\ Martine de Bruijne, ${ }^{3}$ Marian Smeulers ${ }^{4}$
}

To cite: Lansdaal $D$, van Nassau F, van der Steen M, et al. Lessons learned on the experienced facilitators and barriers of implementing a tailored VBHC model in a Dutch university hospital from a perspective of physicians and nurses. BMJ Open 2022;12:e051764. doi:10.1136/ bmjopen-2021-051764

- Prepublication history and additional supplemental material for this paper are available online. To view these files, please visit the journal online (http://dx.doi.org/10.1136/ bmjopen-2021-051764).

Received 18 April 2021 Accepted 07 December 2021

Check for updates

(C) Author(s) (or their employer(s)) 2022. Re-use permitted under CC BY-NC. No commercial re-use. See rights and permissions. Published by BMJ.

For numbered affiliations see end of article.

Correspondence to

Marian Smeulers;

m.smeulers@amsterdamumc.nl

\section{ABSTRACT}

Objective This study aims to obtain insight into experienced facilitators and barriers of implementing a tailored value-based healthcare (VBHC) model in a Dutch university hospital from a perspective of physicians and nurses.

Method A descriptive qualitative study with 12 physicians, nurses and managers of seven different care pathways who were involved in the implementation of a tailored VBHC methodology was conducted. Thematic content analysis was used to analyse the data guided by all factors of the Consolidated Framework for Implementation Research (CFIR).

Findings The method designed for the implementation of a tailored VBHC methodology was appointed as a structured guide for the process. Throughout the implementation process, leadership and team dynamics were considered as important for the implementation to succeed. Also, sharing experiences with other value teams and the cooperation with external Information Technology (IT) teams in the hospital was mentioned as desirable. The involvement of patients, that is part of the VBHC methodology, was considered useful in the decision-making and improvement of the care process because it gave better insights in topics that are important for patients. The time-consuming nature of the implementation process was named as barrier to the VBHC methodology. On top of that, the shaping of the involvement of patients and the ongoing changes in departments were established as difficult. Finally, working with the Electronic Health Records and acquiring the necessary digital skills were considered to be often forgotten and, thus, hindering implementation.

Conclusion Clinical Healthcare organisations implementing a tailored VBHC methodology will benefit from the use of a structured implementation methodology, a well-led strong team and cooperation with (external) teams and patients. However, shaping patient involvement, alignment with other departments and attention to digitisation were seen as a most important concerns in implementation and require further attention.
Strengths and limitations of this study

- The descriptive qualitative design enabled us to explore the experienced barriers and facilitators from the physicians' and nurses' perspective in depth.

- Several teams with different healthcare departments participated, increasing generalisability.

- Not all relevant stakeholders involved in the implementation process, such as patients and managers, were included in this study.

- The study is a single-centre study conducted in one of the largest hospitals in the Netherlands.

\section{INTRODUCTION}

The uneven quality and rising costs of healthcare are a global problem. ${ }^{1}$ In 2006, Porter and Teisberg introduced the management model value-based healthcare (VBHC) to contribute to solving the quality and economic problems in healthcare. ${ }^{12}$ The aim of VBHC is to create value for the patient by improving health outcomes while reducing associated costs. $^{1}{ }^{2}$ The original strategic agenda for value transformation in healthcare, that is, 'value agenda', consist of six elements: (1) organise into Integrated Practice Units, (2) measure outcomes and costs for every patient, (3) move to bundled payments for care cycles, (4) integrate care delivery systems, (5) expand geographic reach and (6) build an enabling information technology platform. ${ }^{2}$ An important aspect of VBHC is the monetary opportunity costs of the entire care process along the patient journey, based on human and material resources used. Consequently, switching focus to health outcomes and care process results in insights that are valuable for reducing practice variation, increasing quality of healthcare and improving costeffectiveness. $^{12}$ 
It is well known that, putting innovative care according to VBHC principles into practice, a major change for the healthcare staff and patients involved is expected. ${ }^{3-8}$ Organising care centred around the patient challenges multidisciplinary professionals from different organisational units to collaborate and coordinate their tasks as well as to share responsibility for continuous healthcare improvement. These are major changes in daily practice that require extensive implementation effort. ${ }^{5}$

Previous studies have found that the model as introduced by Porter and Teisberg ${ }^{2}$ is relevant but incomplete. A reason given for that includes the actual use of outcomes to improve quality of care, which appear not to be sufficiently emphasised. ${ }^{78}$ As such, it is well known that there is a gap between best practice on the one hand, and the actual care performed on the other. ${ }^{89}$ Besides, it is shown in previous research that the use of clinical registration to understand health outcomes in relation to VBHC is desirable. However, it requires leadership at the physician and manager level, should include the opportunity for benchmarking, must contain a well-integrated computerised system and must include a collaborative effort to achieve the best possible way of working. ${ }^{10}$

Implementation within healthcare settings occurs, according to the Consolidated Framework for Implementation Research (CFIR), on different levels; (1) the intervention (2) the individual, (3) the setting within the team ('inner setting'), (4) setting outside the team ('outer setting') and (5) the implementation process. ${ }^{11-14}$ At each level, different facilitating and hindering factors may be experienced by those implementing. First, on the level of the intervention, a very detailed description of the implementation methodology as well as a clear focus on usable tools is considered as helpful. ${ }^{15}$ Second, on the level of the individual involved in the implementation, the success of the implementation depends on the appearance of dedication and intrinsic motivation. ${ }^{12}$ Third, on the level of the inner setting, the productivity within a team will depend on the experienced costs they have to deliver for the result they produce, also in relation to the organisational readiness. ${ }^{16}{ }^{17}$ In line with this, a collaboration between caregivers is a necessity in any healthcare setting to achieve a successful implementation, in which forces are combined. ${ }^{18}$ On the level of the outer setting, cooperation with other teams appears to be beneficial for a successful implementation. ${ }^{11}$ To our knowledge, no study has been published on experienced facilitators and barriers from the perspective of physicians and nurses involved in the implementation of VBHC in a large university hospital as part of a hospital wide structured methodology. Consequently, insight into barriers and facilitators in this regard is needed. ${ }^{1920}$

In the Netherlands, eight University Medical Centers (UMCs) work together to improve the quality of care. Introducing the principles of VBHC is a component of their programme. ${ }^{21}$ The Netherlands Federation of UMCs (NFU) has translated a strategy for the UMCs from Porter's philosophy. ${ }^{22}$ The choice was made to start this strategy with adding value for the patient in the consulting room and to focus on organising care around the patient by working with health-related outcomes. The term 'health outcomes' refers to (1) clinical outcomes, (2) quality of life outcomes (measured by Patient Reported Outcome Measures (PROM)) and (3) patient experiences (measured by Patient Reported Experience Measures; PREM). During consultations, PROM information is used to empower the patient, improve the dialogue, enable shared decision-making (SDM) and tailor care to the patient's needs. A subsequent step is to track costs. The reason costs were added last in this implementation cycle is the underrepresentation of large groups of patients within the UMCs. On top of that, the complex costing of the UMCs and the challenges involved in determining (health related) costs has factored into this. As literature shows, the implementation of VBHC is difficult. ${ }^{3-8}$ Besides, it appears largely unclear how to use PROMs with respect to SDM and the expectations of physicians in that regard. ${ }^{36}$ For implementation to be successful, an understanding of the barriers and facilitators experienced by physicians and nurses is needed. ${ }^{8}$

The Amsterdam UMC has developed its own methodology to implement VBHC according to NFU principles. This strategy is primarily focused on value-driven quality improvement. The approach as made in the Amsterdam UMC is based on elements 1, 2 and 6 of Porter's model. The integrated practice units (IPU's, element 1) is adopted partly and implemented as a multidisciplinary collaboration across specialty-based units within the traditional organisational structure as opposed to Porter's IPU concept that concerns an alternative organisational structure. The reason that element 1 is adopted partly and the other elements of Porter and Teisberg's ${ }^{2}$ model are out of scope, which includes the feasibility and succession of the local improvement cycle in which these elements can be implemented as an extension when opportune. The development of the Amsterdam UMC value-driven quality improvement provided a unique opportunity to study the tailored method of a VBHC implementation from the perspective of physicians and nurses to identify learning points and successes forming a prerequisite for successful implementation in daily practice. The type of VBHC related to this article can also be described as an NFU-based value-driven care programme and, thus, deviated from the original VBHC model of Porter and Teisberg. ${ }^{2}$ The aim of this study was to evaluate facilitators and barriers of implementing a tailored VBHC model in a Dutch university hospital from a perspective of physicians and nurses.

\section{METHOD}

A descriptive qualitative study according to the COREQ (COnsolidated criteria for REporting Qualitative research) checklist was conducted 1 year after the start of the implementation of the tailored model of VBHC within the Amsterdam UMC to better understand and 


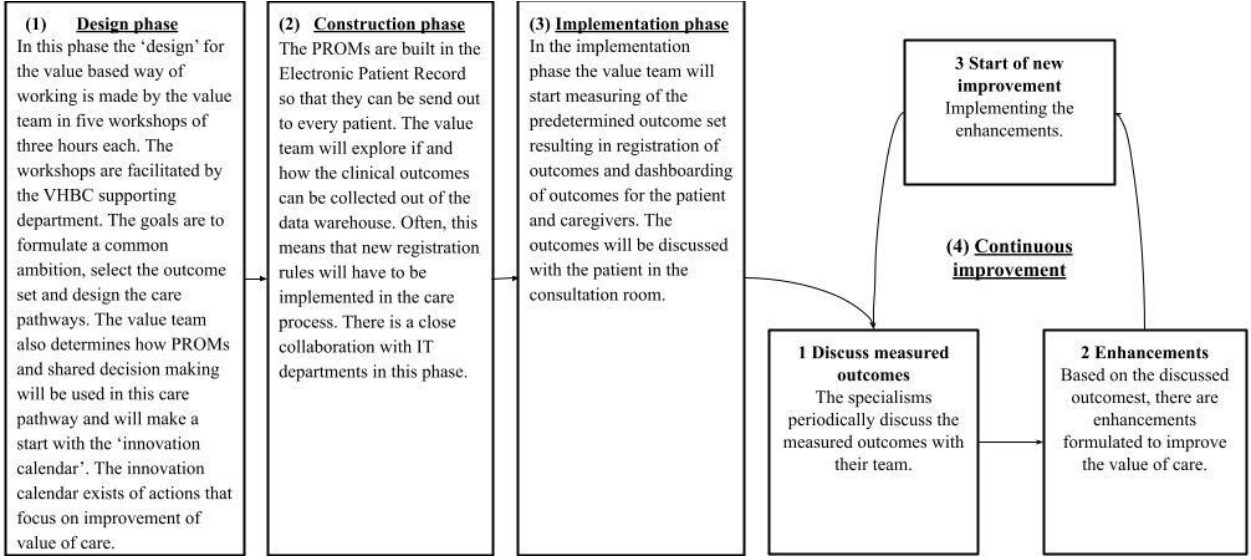

explain representative participants' experienced facilitators and barriers. It is important in this respect that when referring throughout this article to the term 'VBHC', it represents the tailored model specified on the context applicable in the Amsterdam UMC. The research was conducted using semistructured interviews guided by the CFIR framework ${ }^{14}$ because it provides an overview of concepts that guide potential barriers and facilitators of an innovative implementation in healthcare.

\section{Setting and context}

The Amsterdam UMC started in 2017 with the implementation of a VBHC methodology using a locally developed implementation methodology, further referred to as 'VBHC methodology'. In 2019, the Amsterdam UMC originated from two Amsterdam-based university medical centres: the Vrije Universal Medical Center (VUmc) and the Amsterdam Medical Center (AMC). The merger had not yet been completed at the time of the study. The scope of the research includes both locations of the Amsterdam UMC.

\section{Intervention}

The focus of the VBHC methodology as used in the Amsterdam UMC was to achieve continuous improvement based on insight into clinical and patient-reported outcomes and optimisation of the multidisciplinary care process along the patient journey, through a four-phase methodology (see figure 1). First, the goal of value-driven improvement of care must be focused on a systematic approach for value-based quality improvement. Next, value must be integrated within patient communications. Furthermore, the culture of value improvement should be invested in and should include learning platforms for physicians to work with patient outcomes. These four components to work value based should be offered in a strategic agenda. ${ }^{7}$ It is important to note that the methodology included within this article deviates from the VBHC model as designed by Porter and Lee. ${ }^{23}$ This methodology was designed by the strategic team at Amsterdam UMC, specifically applicable to their context. The purpose of the
VBHC methodology designed by the Amsterdam UMC is to give the patient a solid influence on the development and focus that is applied to the relevant clinical condition. Hereby, the methodology seeks to maintain patient values at the highest priority. The patient is present at all sessions in the design phase and is an equal part of the value team. After these sessions, patients are periodically involved in a focus group, patient council or in any other way appropriate to the dynamics of the patient group.

Clinical teams were invited to sign up voluntarily for participation in implementation of the VBHC methodology. Based on the care for a clinical condition, a multidisciplinary 'value team' is created. A clinical lead is appointed to drive the implementation process; this is a physician or nurse working in the clinical team. For each value team a design phase takes place where the team's focus and trajectory are discussed. In this regard, the factors specific to the clinical condition concerned are considered. This process is guided by an external consultant as well as by a specialist of the 'Supporting' department within the hospital. Next, through discussion, a set of outcomes is established representable for the clinical condition and of importance to the patient. Through follow-up sessions, progress is monitored. During implementation, an integral dashboard with outcome and process measures are developed both at the patient and population level to support continuous quality improvement efforts. At the time the research was conducted, none of the teams had fully completed the implementation cycle. The hospital's strategy continuously aims at improving patients' outcomes. In this regard, it has been chosen that within the implementation strategy experiences come first, and when insights have been reached, assessment and improvement of the economic aspect of the VBHC methodology will follow. ${ }^{1}$

\section{Participants}

The programme manager of the tailored VBHC model implementation approached all value teams within Amsterdam UMC to participate in the implementation. 
Participants consisted of physicians, nurses or managers, which possess the role of team member or clinical lead within the implementation of the VBHC methodology. All eight teams that had at least 1 year experience of working with the VBHC methodology were approached for participation. Due to purposive sampling, alongside to increase the credibility and comprehensiveness of the study for data triangulation, the choice was made to have the sampling frame consists of at least one healthcare provider from each of these eight teams.

\section{Patient and public involvement}

No patients were involved in this research.

\section{Data collection}

Data were collected between February 2020 and April 2020. Of the eight teams that were approached, one was not available for participation because of the high workload that existed as a result of the pandemic (COVID-19). To collect data, semistructured interviews were conducted by DL (MSc). Interviews were conducted in person in a restricted area with no other persons present or through a video call. For video calls, Zoom (2011) was chosen to conduct the interviews, because an encrypted connection was possible, and respondents were already familiar with this form of communication. In terms of integrity of the final results the interviewer and respondents did not know each other in advance. Prior to the interview, the respondent's rights and purpose were made known to ensure that the respondent was aware of his/her participation and related responsibilities. Written informed consent was obtained from each participant. Furthermore, in terms of credibility, respondents were informed about the guarantee of confidentiality, the use of their data and that they could withdraw at any time. All the interviews lasted between $30 \mathrm{~min}$ and $60 \mathrm{~min}$ and were audio recorded. Based on the CFIR framework, we developed the interview guide (see online supplemental appendix 1 ). To ensure that the questions were as comprehensible as possible for the respondent, small differences into the questions were commandingly added to the different roles the respondents fulfil (individual clinical leader or operational team member). Due to conformability, a logbook was kept on which adjustments to the topic list were made iteratively, in consideration of the fact that qualitative research can follow new domains and is adaptable. To comply with member check and credibility, every respondent confirmed the correctness of the transcript by e-mail. The transcripts were numbered in order not to make the respondents recognisable to third parties directly. As the data may be used for multiple studies and apply as little interpretation as possible, the choice was made to transcribe the interviews literally. Every audio fragment, transcript and online informed content is stored for 15 years on a secured hard disk of the VUmc. According to the legislation law, ${ }^{24}$ only DL, MvdS and FvN have access to this data.

\section{Data analysis}

A thematic content analysis according to the six steps of Braun and Clarke was used to analyse the data because it allows an interpretation of the participant's meaning of the experienced facilitators and barriers. ${ }^{25}$

After generating and transcribing all interviews, all were read and reread in order to become familiar with the data (step 1). The codes were identified by searching for meaningful units in the interviews labelled by coding words. (Step 2) The analysis of coding words started with deductive coding of the CFIR framework initially or creating a new code when new insights were obtained. To increase the reliability, thematic content analysis was done using Atlas.ti, V.8 (2020) and was cross coded by MvdS and $\mathrm{FvN}$ with consensus in coding. A codebook (see online supplemental appendix 2) was developed to clarify what was meant by a code in order to make it possible for subsequent researchers to apply these coding with the same understanding.

(Step 3) Patterns and themes were searched within the 12 coded interviews in order to understand respondents' experiences. In order to create thematic content, each code was written out, and by sliding codes together, patterns and themes were defined and named. In terms of credibility, MvdS and FvN performed investigator triangulation in terms of recoding the interviews and look for reconciliation and deviation. Given the different contexts in which respondents found themselves, a distinction was made between facilitators and barriers when creating themes given that one concept could be experienced both as a barrier or facilitator. (Step 4) Creating thematic content was evaluated by MvdS. and FvN. (Step 5) The quotes that created the content for each code were structured to theme using Atlas.ti, from which thick descriptions have emerged. ${ }^{25}$

\section{RESULTS}

Six doctors, four nurses and two managers of the seven conducting departments ( $88 \%$ of the total departments that were within the inclusion criteria) participated in this study. The term 'doctor' refers to all types of physicians or medical specialists, such as internists, surgeons, etc. Nine out of 12 interviews took place in person, three interviews took place through video call. Characteristics of the respondents are summarised in table 1.

Participants experienced various barriers and facilitators during the implementation of VBHC. Themes that emerged regarding the methodology were: 'Structured methodology comfortable but difficult in its execution' and 'Involving patients has added value yet challenging'. Themes regarding the implementation process were: 'Team dynamics funds success of the implementation', and 'Collaborations with others motivates'. Finally, 'Applying VBHC in practice' and 'Facilities that are essential for functionality' were themes based on the daily operations of VBHC. Table 2 summarises the 
Table 1 Baseline characteristics of respondents

\begin{tabular}{|c|c|c|c|}
\hline & $\begin{array}{l}\text { Doctors } \\
(n=6)\end{array}$ & $\begin{array}{l}\text { Nurses } \\
(n=4)\end{array}$ & $\begin{array}{l}\text { Managers } \\
(n=2)\end{array}$ \\
\hline \multicolumn{4}{|c|}{ Time working in this function } \\
\hline$<1$ year & 0 & 1 & 0 \\
\hline $2-5$ years & 2 & 1 & 1 \\
\hline 6-10years & 2 & 1 & 0 \\
\hline $11>$ years & 2 & 1 & 1 \\
\hline \multicolumn{4}{|c|}{ Function within the VBHC team } \\
\hline Team member & 0 & 2 & 2 \\
\hline Leader & 6 & 2 & 0 \\
\hline \multicolumn{4}{|c|}{ Duration of the implementation of the VBHC approach } \\
\hline $1-2$ year(s) & 3 & 2 & 0 \\
\hline $3-4$ years & 3 & 2 & 2 \\
\hline
\end{tabular}

VBHC, value-based healthcare.

Table 2 Themes and subthemes for the implementation of the value-based healthcare approach

\begin{tabular}{|c|c|}
\hline Theme & Subtheme \\
\hline $\begin{array}{l}\text { Theme 1: Structured } \\
\text { methodology comfortable but } \\
\text { difficult in its execution }\end{array}$ & $\begin{array}{l}\text { Goal-oriented and } \\
\text { enlightening methodology } \\
\text { Time bound factors } \\
\text { determine implementation } \\
\text { methodology's success }\end{array}$ \\
\hline
\end{tabular}

Theme 2: Involving patients is Patient involvement gave valuable but challenging depth to understanding care paths and needs yet were challenging Macro level patient involvement is challenging

Theme 3: Team dynamics found success of the implementation Forces and concerns within a team Leadership indispensable within a team Ongoing projects distress

Theme 4: Collaboration with externals/others motivates

$\begin{array}{ll}\begin{array}{l}\text { Theme 5: Struggles in } \\ \text { applying VBHC in practice }\end{array} & \begin{array}{l}\text { Dashboards thrill the team } \\ \text { Shared decision-making } \\ \text { complicated in practice } \\ \text { Working with PROMs in daily } \\ \text { practice has advantages }\end{array} \\ \begin{array}{l}\text { Theme 6: Essential for } \\ \text { functionality }\end{array} & \begin{array}{l}\text { Necessities better available } \\ \text { through connection the VBHC } \\ \text { implementation } \\ \text { Digitisation decelerates }\end{array}\end{array}$

PROM, Patient Reported Outcome Measures; VBHC, value-based healthcare. themes and subthemes of experienced barriers and facilitators within the VBHC implementation.

\section{Theme 1: structured methodology comfortable but difficult in} its execution

Subtheme 1.1: goal-oriented and enlightening methodology

According to the majority of respondents, the VBHC implementation methodology provided a structured process that was perceived as clear, goal-oriented and adaptable to one's own situation:

R10: I found it extremely enlightening to see clearly what we were doing and what we ultimately wanted to achieve and how we were going to achieve that with those different steps. That worked in the end (doctor, leader).

Subtheme 1.2: Time bound factors determine implementation methodology's success

Almost all respondents indicated that the VBHC implementation process was time-consuming, they experienced an imbalance between time invested and degree of progress:

R5: We have been working on VBHC for so long now that everyone has a bit of value-driven care tiredness. Like, 'do we have to come together and look at that care path again? We have already done that twenty times'. Yes, it was just like that (nurse, leader).

\section{Theme 2: involving patients has added value, yet is} challenging

Subtheme 2.1: Patient involvement gave depth to understanding care paths and needs yet were challenging

All respondents considered it valuable to involve patients in the VBHC process in order to learn about their experiences within their care process, which gave depth to understanding care paths and needs. Patient counselling during team involvement was deemed necessary in order to clarify the expected role of the patient in the team as well as to support the patient. Patients visiting the hospital once every 3 months in the development of VBHC was associated with burdensome and confrontational, as there is a chance the patient might be confronted with negative impacts of their disease illustrated by other patient stories:

R4: It was also very confronting for the patient, because some people with the disease died when things didn't go well (doctor, leader).

In addition, respondents felt that patients do not need to be involved at every stage of the VBHC methodology, as they are not directly involved in all aspects of healthcare, for example, during the registration facilities in the Electronic Health Record (EHR).

Subtheme 2.2: Macrolevel patient involvement is challenging The competencies of a patient to think on a macrolevel were perceived as a facilitator in the VBHCimplementation 
process because this enabled valuable input about patient values. However, the ability of a patient to represent the patient population as a whole was perceived as more difficult than expected.

R3: I think we have to be realistic, that $98 \%$ of the patients are not suitable to think 'Well, we have a group of 1500 patients', so we need someone who doesn't just think her own way, it's also about being able to think on a population level (Nurse, leader).

By extension, it was indicated that the way in which patients were involved was also difficult, for example, the amount of guidance and the role a patient take on. If there was no standard method for involving patients, it was indicated that the structure of patient involvement had to be discussed in detail

\section{Theme 3: Team dynamics funds success of the implementation}

\section{Subtheme 3.1: forces and concerns within a team}

Driven individual team members as well as an enthusiastic team that had a bond of trust and pursue, the same goal was considered as beneficial to the implementation. In addition, a small team was also named as a facilitator because this made it easier to discuss changes:

R10: Another important aspect is forming a strong team... ... and that helped a lot, really. You have the same goal. We all work with the same tool. So that's an advantage (doctor, leader).

Several respondents indicated that sufficient time for healthcare professionals was essential to get used to changes, such as working with PROM questionnaires and SDM. However, sufficient progress within the implementation of the VBHC methodology was also named as important, to avoid losing motivation, referred to as 'you lose momentum'.

\section{Subtheme 3.2: Leadership indispensable within a team}

Leadership in a team was expressed as crucial to realise the VBHC implementation by all respondents. Various characteristics of a successful leader were addressed: someone who (continues to) take people along in the VBHC implementation during the entire process in order to keep other team members on board as well as someone who collects and shares information:

R6: You need a leader to keep pushing the team. Who says, "Let's do it this way," and comes to give information (nurse, team member).

It was noted that it is important for the leader to be able to recognise and understand practical situations. This makes occurred problems during the implementation of the VBHC methodology easier to understand:

R2: You need people who know what's going on and are involved within the clinic (doctor, leader).
Subtheme 3.3: Ongoing projects distress

Other projects that were introduced during the VBHC implementation were seen as a barrier because it frustrated that build up of VBHC was disrupted. An example of this is the ongoing merger between the two locations of the hospital, which took a lot of time and effort to (re) build mutual trust, knowledge and (policy) attention:

R4: Because we worked a long time to get that dashboard operational, we presented it to the staff "..." and we moved to the other hospital there was nothing left anymore (doctor, leader).

\section{Theme 4: Collaboration with externals/others motivates}

\section{Subtheme 4.1: Collaborative effort useful}

Sharing experiences and best practices with other value teams was perceived as a facilitator for the implementation of VBHC because teams can benefit from each other's lessons learnt:

R8: I might want to talk to a larger team myself. So, more the connection with other teams, but also with my own value team."...”The contact may be slowing down the implementation process, but I think that there could have been other solutions in terms of content, rather than that I have invented it myself (doctor, leader).

\section{Theme 4.2: supporting team intended}

The participation of a representative team member from the central VBHC support team within the hospital was regarded as a facilitator in order to provide knowledge of VBHC implementation, competence development, enthusing, thinking about potential facilitators and barriers and coming up with new ideas:

R4: I've really had a lot of support of (name VBHC member of support team). It has helped in making appointments, always came up with new ideas, or if the team was not happy for a moment she came up with a nice idea (doctor, leader).

The support of the board of directors was not always noticed by the respondents. Prioritisation for accomplishing the VBHC implementation were identified as focal points of daily policy:

R9: The implementation does have the attention, but it is not prominent in any particular policy line or structure embedded in the outpatient care That frustrates (manager, team member).

Subtheme 4.3: Collaboration with data support brings comfort but frustrates process

Not only did all respondents indicate that there was a strong sense of dependency between teams and data support because of their important position within the VBHC implementation, they also mentioned a lack of understanding of mutual expectations. The low delivery 
frequency of improved technology due to capacity problems was described as a negative influence for continuous improvement:

R6: What happened now, you're a value group, you have a question, ask your question, but I just have to hope I'm on a list, and I just have to hope something is being built somewhere,"... "If this is what we think is important to each other, you have to make sure that your whole system is actively involved in the care you provide (nurse, leader).

\section{Theme 5: Struggles in applying VBHC in practice}

\section{Subtheme 5.1: Dashboards thrill the team}

Several respondents indicated that there was willingness to register outcomes in daily practice, allowing use for benchmark and improvement. This is related to the fact that several respondents described that working with outcomes can motivate in daily practice and could create continuous improvement within a team:

R7: It shows what measurement we're missing for a patient, and that kind of measurements makes you very motivated to say, "hey, we're going to have to set it up better (Nurse, team member).

Working with outcomes was also seen by leaders as an opportunity for nurses to innovate and differentiate their competencies. Yet, nurses, who were not leaders of a value team themselves, did not indicate this.

It was mentioned by several respondents that the lack of adequate registration within the EHR was caused by difficulty adapting their daily working process with registrations, due to the non-user friendly EHR system. Several respondents also indicated that when the PREMs and PROMs are discussed within the value teams, this can be perceived as confrontational, disturbing and/or as a violation of the employee's privacy by colleagues:

R3: They'll suddenly be told, that based on measured outcomes, 'maybe you should plan less visits', or 'you'll have to be a little more effective with the appointments'. So, it can still be experienced as a kind of intrusion, which can be complicated (nurse, leader).

\section{Subtheme 5.2: SDM complicated in practice}

In daily practice, the VBHC implementation aimed to find a balance for SDM and the possibilities thereof. In the context of SDM, it is in fact desirable to let the patient's preference become a reality. However, it was experienced by caregivers that the patient's preference does not always correspond to the oath of caregivers to provide what they consider to be the best care. On top of that, organisational culture was described as a barrier in the success of the VBHC implementations' SDM. It was mentioned as difficult from a culture perspective to let patients take the lead:
R6: There is also an aspect of culture certainly in the surgical doctors that they know what's good for the patient. So, you can soon have all your data to be able to decide together, but if you still think, as a doctor, that your opinion is worth more than that of your patient, then yes, that is not the goal of the implementation (manager, team member).

\section{Subtheme 5.3: Working with PROMs in daily practice has advantages}

The use of PROMs in practice was indicated as important, because it allowed the quality of life to be properly measured and discussed with the patient. However, the majority of the respondents indicated that usage of PROMs took time to put into practice, because healthcare providers were not used to working with questionnaires in daily practice and had difficulty incorporating this in their outpatient visit work process:

R2: We are used to questionnaires for scientific research, and not at all for daily clinic"... "there was room for improvement (nurse, leader).

\section{Theme 6: Essentials for functionality}

Subtheme 6.1: Necessities for value teams from hospital board better available through connection the VBHC implementation It was indicated that availability of funding and financing for the VBHC process improvement could be motivational to start the implementation. Almost all respondents indicated that, despite the length of the implementation period, their intrinsic motivation for implementation was maintained because of the (continued) recognition of usefulness of VBHC. However, if the importance of implementation was no longer seen, neither was the need to devote effort to VBHC implementation:

R1: I don't see the immediate interest anymore, and then I prefer to continue with my own research (doctor, leader).

\section{Subtheme 6.2: Digitisation decelerates}

Almost all respondents indicated that digitisation was perceived as a barrier, despite the awareness that it can help in daily practice. The healthcare sector was mentioned by some respondents as not completely designed to measure reliable outcomes, for example, with comorbidity, which could result in registration resistance. A few respondents named that the impact of digitisation skills was felt as 'forgotten' in the VBHC implementation. They felt it was assumed that every healthcare provider knows how to work with computers, when this is not always the case:

R6: They suddenly assume that a caregiver can work with a computer and talk to the patient. We find that quite normal but it's not even the easiest thing for my generation who are fortysomething because we weren't born with the phone, and the computer 
didn't come into my life until I was twenty (manager, team member).

\section{DISCUSSION}

This study identified the facilitators and barriers experienced by physicians and nurses within the implementation of a tailored VBHC methodology in a Dutch university hospital. We identified six themes: (1) The locally developed VBHC methodology was experienced as a facilitator by the teams because it gave structure but was also perceived as time-consuming, (2) Involvement of patients was indicated as valuable because patients gave depth to understanding care paths and needs. However, frequency of patient participation, design of patient involvement, representativeness and confrontational aspect of patient involvement were perceived as challenging, (3) For functioning and dynamicity within a team, several points were indicated as facilitators of the implementation, namely; having a bond of trust, presence of goal-orientation, having a small team, sufficient time to adjust the way of working and leadership. Other projects that were introduced simultaneously to the VBHC implementation were perceived as a barrier in this regard. (4) Cooperation with other (value, Information Technology and supporting) teams and prioritising of board of directors were indicated as motivational factors for the execution of implementation. On the other hand, lack of mutual understanding between these teams was perceived as a barrier in the implementation process. (5) Adaptation to working with SDM and PROMs in daily practice were desired but raised issues with breaking through culture and could be considered as confrontational when results of one's own medical practices become insightful. Finally, (6) Funding for the VBHC implementation and continued recognition of the usefulness of the VBHC implementation was perceived as a facilitator the implementation. Obtaining and maintaining digitisation and gathering skills to work with electronic health systems were felt as much forgotten during VBHC implementation.

The literature shows that there are still insufficient in-depth insights into which factors are promoting or hindering during implementation. ${ }^{9}$ This also emerged from our research, where insight into the perceived barriers can be conducive to bypassing hindering factors in the implementation process. When planning complex changes in practice, potential barriers at various levels need to be addressed. Planning needs to take into account the nature of the innovation; characteristics of the professionals and patients involved; and social, economic and political context.

Nilsson and Sandoff stated that the 'voice' of the caregiver outweighs the voice of the patient in his involvement in the implementation of VBHC. ${ }^{26}$ This was also echoed in our study, where stakeholders mentioned that it was difficult from a culture perspective to empower and involve patients in the tailored VBHC implementation.
This was also hampered by a lack of knowledge on how to involve patients in the implementation process. For example, not all healthcare providers were satisfied with the clarity of the expected patient's role in the tailored VBHC methodology and the probability that the patients' perspective did not apply to the entire population. In order to implement the tailored VBHC methodology in a sustainable manner, it could be recommended to care providers to consult with each other about the type of design, in which patients will be involved in the implementation to overcome this barrier. With this reason, it is important to investigate with both caregivers and patients whether, and in what way, they can and want to participate best in these designs.

Within the implementation of VBHC, respondents of our study experienced the registration of outcomes in the EHR as a barrier because the electronic systems do not include an intuitive visualisation of the care provider, nor the ability to make quick adjustments. ${ }^{20} 2728$ However, it was also mentioned that working with technology appears to be facilitating during the implementation of VBHC by the willingness to benchmark outcomes and be able to compare and improve with other (value) teams. ${ }^{17}$ Therefore, it is important to continue to support working with outcomes for comparability among hospitals. ${ }^{29}$ Follow-up research should be oriented towards how to properly facilitate EHR registration in daily practice. As a result, data can be better used to measure outcomes and facilitate the possibility of benchmarking. The workflow in the EHR should be logical in relation to daily care and should not take additional time.

The required behavioural and cultural change among healthcare providers to adjust the provided care were identified as barriers to the implementation of VBHC. ${ }^{11}$ 30-33 For example, the process of using PROM questionnaires was experienced as difficult, because healthcare providers are often not used to working with these questionnaires in daily practice. Besides, sharing and discussing outcomes of individual healthcare professionals in order to improve practice were perceived as confronting, difficult and harmful for team dynamics. This current finding has not come to light in comparable studies, which provides new insight. An explanation for the confrontational matter may be caused by a lack of mutual trust or unease with the confrontation of individual healthcare providers' results. The creation of a culture that guarantees safety and is characterised by continuous improvement is of great importance. Respondents identified that discussing how to understand each other's value team-related health outcomes, and letting each other learn from one another's mistakes, can be brought as an improvement strategy instead of a threat. This also coincides with the fact that a patient never has only one treating healthcare professionals, but several, and the outcome cannot be related to the work of one physician. Therefore, guidance to enable changes in day-to-day work that healthcare providers have to endure will be beneficial for the implementation of VBHC. 
A major strength in this study is the descriptive qualitative design, which has enabled us to explore the experienced barriers and facilitators from different points of view. To our knowledge, no studies have been published about representatives of project teams' experienced facilitators and barriers of implementing VBHC for university hospitals in the Netherlands. Therefore, our results contribute to the body of knowledge on this topic. In addition, a partially deductive analysis was executed, based on the CFIR framework, which resulted in a more comprehensive analysis since new insights were also found in addition to the themes represented in the model. The main limitation in this study is that patients could not be interviewed, nor all value teams could be included or refrained from participation due to inaccessibility, as a result of the COVID-19 pandemic. As a result, there was a selection in the departments with the lowest work pressure and an incomplete representation of the experienced facilitators and barriers of the stakeholders involved in the implementation. For this reason, it is important in follow-up research that patients are included, and additional attention is devoted to a representative selection of respondents from each participating value team. Besides, adding quantitative analysis to make findings comparable is necessary to reach a further conclusion in this matter.

\section{CONCLUSION}

In summary, this descriptive qualitative research contributes to giving insight and a preliminary impression into the experienced facilitators and barriers from the perspective of physicians and nurses to the implementation of the tailored VBHC methodology in a Dutch university hospital. The most influential facilitating factors to the implementation process that were found in our study were the use of a structured implementation methodology, the presence of a well lead, strong, team and the incentive to improve care by registering outcomes in order to create benchmarking. A prerequisite for successful implementation is sufficient communication, prioritisation and managing expectations between a value team and other (value, data, Board of Directors and supporting) teams. However, barriers were experienced regarding the design of patient involvement, confrontational aspect of insight into outcomes and usage of the EHR in daily practice. These issues require more proactive attention and engagement of professionals and patients from the start and, thereafter, for instance, by sharing experiences among value teams.

\section{Author affiliations}

${ }^{1}$ Strategy and Improvement, Amsterdam UMC De Boelelaan Site, Amsterdam, The Netherlands

${ }^{2}$ Vrije Universiteit Amsterdam, Department of Public and Occupational health, Amsterdam Public Health Institute, Amsterdam University Medical Centres, Amsterdam, The Netherlands

${ }^{3}$ Department of Public and Occupational Health, Amsterdam UMC Location VUmc, Amsterdam, The Netherlands
${ }^{4}$ Division of Outpatient Department, Amsterdam UMC Locatie AMC, Amsterdam, North Holland, The Netherlands

Acknowledgements The authors would like to thank the value teams who participated in the study.

Contributors Each author has taken sufficient part in the work to assume public responsibility for suitable parts of the content, and all the conditions of authorship have been met. Each author has seen and accepted the submitted manuscript's content. More specifically, DL contributed to the study planning, the study concept and design, obtaining the data of the study, the analysis and the interpretation of the data. Besides, the drafting, revising the article, final approval and submitting of the article was also done by DL. The other four authors were always included in updated versions of the paper, using their expertise to put down improvements as best as possible. FvN and MvdS contributed to the study design, acquisition of the data, interpretation of the data, revising the article and final approval of the submitted article. MvdS contributed to this study concept and design by defining the tailored VBHC methodology on which this study was based and approval of the submitted article. MdB contributed interpretation of the data, revising the article and approval of the submitted article. All authors read and approved the final manuscript. Non-author contributors: The Value-Driven Care Approach strategic team contributed to the concept of the study. MS is the guarantor.

Funding The authors have not declared a specific grant for this research from any funding agency in the public, commercial or not-for-profit sectors.

Competing interests None declared.

Patient consent for publication Not applicable.

Ethics approval An ethical approval has not been considered necessary for interviewing medical doctors by the Ethical Review Committee of the Amsterdam UMC with number W20_102\#20.129, according to the Dutch Medical Ethics Law.

Provenance and peer review Not commissioned; externally peer reviewed.

Data availability statement Data are available upon reasonable request. The data are available by e-mail upon request.

Supplemental material This content has been supplied by the author(s). It has not been vetted by BMJ Publishing Group Limited (BMJ) and may not have been peer-reviewed. Any opinions or recommendations discussed are solely those of the author(s) and are not endorsed by BMJ. BMJ disclaims all liability and responsibility arising from any reliance placed on the content. Where the content includes any translated material, BMJ does not warrant the accuracy and reliability of the translations (including but not limited to local regulations, clinical guidelines, terminology, drug names and drug dosages), and is not responsible for any error and/or omissions arising from translation and adaptation or otherwise.

Open access This is an open access article distributed in accordance with the Creative Commons Attribution Non Commercial (CC BY-NC 4.0) license, which permits others to distribute, remix, adapt, build upon this work non-commercially, and license their derivative works on different terms, provided the original work is properly cited, appropriate credit is given, any changes made indicated, and the use is non-commercial. See: http://creativecommons.org/licenses/by-nc/4.0/.

ORCID iD

Dane Lansdaal http://orcid.org/0000-0002-0139-5564

\section{REFERENCES}

1 Porter ME, Lee TH. The strategy that will fix health care. Harv Bus Rev 2013.

2 Porter ME, Teisberg EO. Redefining Health Care - Creating ValueBased Competition. Boston: Harvard Business School Press, 2006.

3 Spatz ES, Elwyn G, Moulton BW, et al. Shared decision making as part of value based care: new U.S. policies challenge our readiness. Z Evid Fortbild Qual Gesundhwes 2017;123-124:104-8.

4 Foster A, Croot L, Brazier J, et al. The facilitators and barriers to implementing patient reported outcome measures in organisations delivering health related services: a systematic review of reviews. $J$ Patient Rep Outcomes 2018;2:46.

5 Damman OC, Jani A, de Jong BA, et al. The use of PROMs and shared decision-making in medical encounters with patients: an opportunity to deliver value-based health care to patients. J Eval Clin Pract 2020;26:524-40.

6 van der Nat PB, Nat PBVder. The new strategic agenda for value transformation. Health Serv Manage Res 2021:095148482110117. 
7 van Veghel D, Daeter EJ, Bax M, et al. Organization of outcomebased quality improvement in Dutch heart centres. Eur Heart J Qual Care Clin Outcomes 2020;6:49-54.

8 Grol R, Wensing M. What drives change? barriers to and incentives for achieving evidence-based practice. Med $\mathrm{J}$ Aust 2004;180:S57-60.

9 Kampstra NA, Zipfel N, van der Nat PB, et al. Health outcomes measurement and organizational readiness support quality improvement: a systematic review. BMC Health Serv Res 2018;18:1-14.

10 Nilsson $\mathrm{K}$, Bååthe $\mathrm{F}$, Andersson $\mathrm{AE}$, et al. Experiences from implementing value-based healthcare at a Swedish University Hospital - an longitudinal interview study. BMC Health Serv Res 2017;17:169.

11 Hellström A, Lifvergren S, Gustavsson S, et al. Adopting a management innovation in a professional organization. Business Process Management Journal 2015;21:1186-203.

12 Abdallah A. Implementing quality initiatives in healthcare organizations: drivers and challenges. Int J Health Care Qual Assur 2014;27:166-81 http:// dx.doi.org/

13 Damschroder LJ, Reardon CM, Lowery JC. The consolidated framework for implementation research (CFIR). In: Handbook on implementation science. Edward Elgar Publishing, 2020.

14 van den Heuvel J, Does RJMM, Bogers AJJC, et al. Implementing six sigma in the Netherlands. Jt Comm J Qual Patient Saf 2006;32:393-9.

15 Gray JAM. Better value healthcare--the 21st century agenda. $Z$ Arztl Fortbild Qualitatssich 2007;101:344-6.

16 Zipfel N. Beyond Value-Based Health Care. How to use outcome measurement to improve quality of care in heart care?(Doctoral dissertation,[SI: sn]). 2020.

17 Matziou V, Vlahioti E, Perdikaris P, et al. Physician and nursing perceptions concerning interprofessional communication and collaboration. J Interprof Care 2014;28:526-33.

18 Papanicolas I, Woskie LR, Jha AK. Health care spending in the United States and other high-income countries. JAMA 2018;319:1024-39.

19 Hijden Evander, Steenhuis S, Hofstra G. Ontwikkelingen in zorginkoop: van inkoop van verrichtingen naar inkoop van zorgbun dels. Achtergrond, contractelementen en impact voor zorgaanbieders. Maandblad voor Accountancy en Bedrijfseconomie, 93(5/6), 2020

20 Weert Nvan, Hazelzet J. Gepersonaliseerde zorg ALS waardegedreven zorg. Qroxx, 2020. Available: https://www.qruxx. com/gepersonaliseerde-zorg-als-waardegedreven-zorg/

21 NFU (z.j.). Waardegedreven Zorg position paper. Available: https:// nfukwaliteit.nl/pdf/NFU-Position_Paper_Waardegedreven_Zorg.pdf

22 Amsterdam UMC. Waardegedreven zorg binnen Het Amsterdams UMC. Aanpak waardetuinen, 2017.

23 Porter ME, Lee TH. The strategy that will fix health care. Harv Bus Rev 2013;91:24.

24 World Medical Association. World Medical association Declaration of Helsinki: ethical principles for medical research involving human subjects. JAMA 2013;310:2191-4.

25 Braun V, Clarke V. Using thematic analysis in psychology. Qual Res Psychol 2006;3:77-101.

26 Nilsson K, Sandoff M. Leading implementation of the management innovation value-based healthcare at a Swedish university hospital. $J$ Hosp Adm 2017;6:51.

27 Basch E. Patient-Reported Outcomes - Harnessing Patients' Voices to Improve Clinical Care. N Engl J Med Overseas Ed 2017;376:105-8.

28 Porter ME, Baron JF, Chacko JM. The UCLA medical center: kidney transplantation, 2010.

29 Kelley TA. International Consortium for health outcomes measurement (ICHOM). Trials 2015;16:04.

30 Unit El. Value-Based healthcare: a global assessment. The Economist. Recuperado em 2016;22:2018.

31 de Koeijer R, Hazelzet J. Wat is nodig voor duurzame implementatie van value based healthcare? Tijdschrift voor Arbeidsvraagstukken 2017;33.

32 Veld M, Paauwe J, Boselie P. Hrm and strategic climates in hospitals: does the message come across at the ward level? Human Resource Management Journal 2010;20:339-56.

33 Radnor ZJ, Holweg M, Waring J. Lean in healthcare: the unfilled promise? Soc Sci Med 2012;74:364-71. 